\title{
Aging in a Two-Dimensional Ising Model with Dipolar Interactions
}

\author{
Julio H. Toloza, Francisco A. Tamarit, Sergio A. Cannast \\ Facultad de Matemática, Astronomía y Física, Universidad Nacional de Córdoba, Ciudad Universitaria, 5000 Córdoba, \\ Argentina
}

(February 13, 2018)

\begin{abstract}
Aging in a two-dimensional Ising spin model with both ferromagnetic exchange and antiferromagnetic dipolar interactions is established and investigated via Monte Carlo simulations. The behaviour of the autocorrelation function $C\left(t, t_{w}\right)$ is analyzed for different values of the temperature, the waiting time $t_{w}$ and the quotient $\delta=J_{0} / J_{d}, J_{0}$ and $J_{d}$ being the strength of exchange and dipolar interactions respectively. Different behaviours are encountered for $C\left(t, t_{w}\right)$ at low temperatures as $\delta$ is varied. Our results show that, depending on the value of $\delta$, the dynamics of this non-disordered model is consistent either with a slow domain dynamics characteristic of ferromagnets or with an activated scenario, like that proposed for spin glasses.
\end{abstract}

PACS numbers: 75.40.Gb, 75.40.Mg, 75.10.Hk

Microscopic long ranged interactions are always of interest in different fields of physics because they can give rise to a variety of unusual macroscopic behaviours. Perhaps the better example in condensed matter are dipole-dipole interactions. In particular, the competition between long-range antiferromagnetic dipolar interactions and short-range ferromagnetic exchange interactions can give rise to several interesting magnetic phenomena. Recent works in two dimensional uniaxial spin systems, where the spins are oriented perpendicular to the lattice and coupled with these kind of interactions, have shown a very rich phenomenological scenario concerning both its equilibrium statistical mechanics 1 . interest due to aspects related to information storage in ultrathin ferromagnetic films. Moreover, there are several contexts in which a short-ranged tendency to order is perturbated by a long-range frustrating interaction. Among others, model systems of this type has been propeffed to study avoided phase transitions in supercooled liquids 6 and charge density waves in doped antiferromagnets $\mathbf{b}$ 6.

The above mentioned systems can be described by an Ising like Hamiltonian of the type

$$
H=-J_{0} \sum_{<i, j>} \sigma_{i} \sigma_{j}+J_{d} \sum_{(i, j)} \frac{\sigma_{i} \sigma_{j}}{r_{i j}^{3}}
$$

where the spin variable $\sigma_{i}= \pm 1$ is located at the site $i$ of a square lattice, the sum $\sum_{<i, j>}$ runs over all pairs of nearest neighbor sites and the sum $\sum_{(i, j)}$ runs over all distinct pair of sites of the lattice; $r_{i j}$ is the distance (in crystal units) between sites $i$ and $j ; J_{0}>0$ and $J_{d}>0$ are the ferromagnetic exchange and antiferromagnetic dipolar coupling parameters respectively. For simplicity, we rewrite this Hamiltonian as follows:

$$
H=-\delta \sum_{<i, j>} \sigma_{i} \sigma_{j}+\sum_{(i, j)} \frac{\sigma_{i} \sigma_{j}}{r_{i j}^{3}}
$$

with $\delta=J_{0} / J_{d}$. There are few numerical results concerning the equilibrium statistical mechanics, i.e. the finite temperature phase diagram, of this model. In a recent work MacIsaac and coauthors 3 have shown that the ground state of Hamiltonian (2) is the antiferromagnetic state for $\delta<0.85$. For $\delta>0.85$ the antiferromagnetic state becomes unstable with respect to the formation of striped domain structures, that is, to state configurations with spins aligned along a particular axis forming a ferromagnetic strip of constant width $h$, so that spins in adjacent strips are anti aligned, forming a super lattice in the direction perpendicular to the strips. They also showed that striped states of increasingly higher thickness $h$ becomes more stable as $\delta$ increases from $\delta=0.85$. Moreover, they showed that the striped states are also more stable than the ferromagnetic one for arbitrary large values of $\delta$, suggesting such a phase to be the ground state of the model for $\delta>0.85$. Monte Carlo calculations on finite lattices at low temperature 3 , gave further support to this proposal, at least for intermediate values of $\delta$. Furthermore, such simulations have shown that striped phases of increasingly higher values of $h$ may become thermodynamicatly stable at finite temperatures

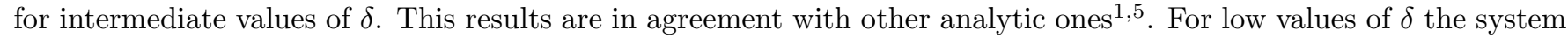
presents an antiferromagnetic phase at low temperatures. At high temperatures, of course, the system becomes paramagnetic.

The dynamics of the model is characterized by the formation and growth of magnetic domains, due to the competition between the exchange and the dipolar interactions, which at low temperatures generate very large relaxation times. In a Monte Carlo study of the time evolution of the magnetization, Sampaio et al have shown the existence of 
two different type of relaxation, according to the value of $\delta$. For $\delta>\delta_{c} \sim 2.7$ the magnetization relaxes exponentially, with a relaxation time that depends both on the temperature and $\delta$. For $\delta<\delta_{c}$ the magnetization presents a power law decay, with an exponent independent of $\delta$. They also showed the existence of strong hysteresis effects in the presence of an external magnetic field for $\delta>\delta_{c}$. Since the relaxation time in this case increases with $\delta$, the area of the hysteresis loops increases with $\delta$. Hysteresis can also be detected in a simulation by looking at the evolution of the system starting from different initial configurations. In Fig. 1 we show snapshots of the evolution of a $N=64 \times 64$ system and $\delta=4$. For $\delta<\delta_{c}$ hysteresis effects becomes very small and the magnetization presents a quasi linear dependence on the magnetic field, up to the saturation field (see Fig.2). Hysteresis is a typical non-equilibrium phenomenon associated to domain dynamics with large relaxation times. In this scenario one also expects the presence of aging effects, that is, history-dependence in the time evolution of the response functions after the system has been quenched into some non-equilibrium state.

Aging phenomena has been vastly studied in disordered systems such as spin glasses (see Ref. 8 and references therein), which are essentially out of equilibrium on experimental timssales. However, they appear also in the phase ordering kinetics of ordered systems, such as the Ising ferromagnet 10.11 , associated with a slow domain dynamics, Aging can be observed in real systems through different experiments. A typical example is the zero-field-cooling 12 experiment, in which the sample is cooled in zero field to a sub-critical temperature at time $t_{0}$. After a waiting time $t_{w}$ a small constant magnetic field is applied and subsequently the time evolution of the magnetization is recorded. It is then observed that the longer the waiting time $t_{w}$ the slower the relaxation.

Although aging can be detected through several time-dependent quantities, a straightforward way to establish it in a numerical simulation is to calculate the spin autocorrelation function

$$
C\left(t, t_{w}\right)=\frac{1}{N} \sum_{i}\left\langle\sigma_{i}\left(t+t_{w}\right) \sigma_{i}\left(t_{w}\right)\right\rangle
$$

where $\langle\cdots\rangle$ means an an average over different realizations of the thermal noise and $t_{w}$ is the waiting time, measured from some quenching time $t_{0}=0$.

In this work we present the results of Monte Carlo simulations in the two-dimensional Ising spin model defined by the Hamiltonian (2) on a $N=20 \times 20$ square lattice with free boundary conditions. We chose the heat-bath algorithm for the spin dynamics and time is measured in Monte Carlo steps per site. The quantity (3) is averaged over 100 samples; for each run the system is initialized in a random initial configuration corresponding to a quenching from infinite temperature to the temperature $T$ at which the simulation is done. We analyze the behaviour of $C\left(t, t_{w}\right)$ as a function of the observation time $t$, for different values of $t_{w}, \delta$ and $T$.

At enough high temperatures we find that the system does not present aging, that is, for any value of $\delta$ there is a temperature above which $C\left(t, t_{w}\right)$ is independent of $t_{w}$, as expected in a paramagnetic phase. At low temperatures we find different types of aging behaviours as $\delta$ is varied.

The typical behaviours of $C\left(t, t_{w}\right)$ are illustrated in Figures 3 and 4 , for $T=0.5$ and different values of $\delta$ (waiting times $\left.t_{w}=5^{n}(n=2, \ldots, 6)\right)$. We also analyzed, for the same values of $T$ and $\delta$, the time evolution of the magnetization per site $m(t)$ and the staggered magnetization per site $m_{s}(t)$ starting from different initial conditions , in order to characterize the different relaxation regimes.

In Fig.3a we see the typical behaviour of $C\left(t, t_{w}\right)$ in the antiferromagnetic state. The characteristic signature of $C\left(t, t_{w}\right)$ in this regime is the appearance of a plateau at some intermediate value of $t$ independent of $t_{w}\left(t \sim 10^{3}\right.$ for $\delta=-1)$, where $C\left(t, t_{w}\right)$ remains constant for a period of time that depends on $T$ and $\delta$; after such period $C\left(t, t_{w}\right)$ relaxes to zero. We also see a dependency on $t_{w}$, that is, aging. The inset shows the evolution of $m(t)$ (filled circles) and $m_{s}(t)$ (open circles) starting from a random initial condition. We also analyzed the evolution of the same quantities starting from a fully magnetized initial state $(m(0)=1)$. We did not find any hysteresis effect in this region, the only difference being a much more slower convergence of $m_{s}(t)$ towards a constant value. These kinds of behaviours appear for negative values and also for small positive valyes of $\delta$ at enough small temperatures, in agreement with previous calculations of the phase diagram of the model2. The existence of these plateaus suggests some type of quasiequilibrium state. This behaviour is rather unusual in this kind of system and it is probably related to the long range character of the interactions.

In Fig.3b we see the typical behaviour of $C\left(t, t_{w}\right)$ in the paramagnetic state, where it does not depend on $t_{w}$. In this case both $m(t)$ and $m_{s}(t)$ converge fast to zero, independent of the initial configuration, as expected. This result also illustrates the characteristic shape of the low temperature phase diagram of the model (see Fig.6 of Ref.2): following a line of constant temperature in the $(\delta, T)$ diagram one can go from the antiferromagnetic region for low values of $\delta$ to the striped one for high values of $\delta$, passing through a paramagnetic region at some intermediate values of $\delta$. For low enough temperatures it is expected a transition line between the antiferromagnetic and the striped regions, without an intermediate paramagnetic one. It is worth to note the logarithmic decay of $C\left(t, t_{w}\right)$. This is a characteristic feature of this dynamics, that is, the relaxation of $C\left(t, t_{w}\right)$ is always slow, even in the paramagnetic region. 
In Fig. 4 we show $C\left(t, t_{w}\right)$ for different values of $\delta$ corresponding to the striped region, below 3 (figures $4 \mathrm{a}$ and $4 \mathrm{~b}$ ) and above (figures $4 \mathrm{c}$ and $4 \mathrm{~d}$ ) $\delta_{c} \sim 2.7$. The behaviour of $m_{s}(t)$ in all these cases shows no traces of antiferromagnetic ordering. On the other hand, the behaviour of $m(t)$ starting from a random $(m(0)=0)$ and a fully magnetized $(m(0)=1)$ states shows a clear differentiation between both regions: while for $\delta>\delta_{c}$ strong hysteresis effects appear (see also Fig.1), for $\delta<\delta_{c}$ such effects are negligible, in agreement with the results of Ref 3 (see also Fig.2).

We found that $C\left(t, t_{w}\right)$ obeys a different type of dynamic scaling law for every one of the two striped regions, as can be seen from the data collapse of figure 5 . For $\delta<\delta_{c} C\left(t, t_{w}\right)$ obeys the dynamic scaling

$$
C\left(t, t_{w}\right) \propto c_{\delta}\left(\log (t) / \log \left(\tau\left(t_{w}\right)\right)\right)
$$

(see figures $5 \mathrm{a}$ and $5 \mathrm{~b}$ ), while for $\delta>\delta_{c}$ it obeys the following scaling

$$
C\left(t, t_{w}\right) \propto c_{\delta}\left(t / \tau\left(t_{w}\right)\right)
$$

(see figures $5 \mathrm{c}$ and $5 \mathrm{~d}$ ), where the time scale $\tau\left(t_{w}\right) \mathrm{B}$ is shown in the insets. We observed, for values of $\delta$ far enough of $\delta_{c}$, the scaling form $\tau\left(t_{w}\right) \sim t_{w}^{a}$. The log-log linear fitting from Fig.5 gives the following values: $a=0.83 \pm 0.02$ for $\delta=2.0 ; a=0.93 \pm 0.05$ for $\delta=3.5$ and $a=1.00 \pm 0.05$ for $\delta=4.0$. This values implies for $\delta$ well below $\delta_{c}$ a logarithmic scaling $C\left(t, t_{w}\right) \propto c\left(\log (t) / \log \left(t_{w}\right)\right)$ (Fig.5a). The scaling form of $C\left(t, t_{w}\right)$ deviates from the previous one as $\delta$ approaches $\delta_{c}$ (Fig.5b). For $\delta>\delta_{c}$ we observe a scaling form $C\left(t, t_{w}\right) \propto c\left(t / t_{w}^{a}\right)$ (figures $5 \mathrm{c}$ and $5 \mathrm{~d}$ ), with a exponent $a$ that approaches the unity as $\delta$ increases.

It has been proposed 141 that aging phenomena are based on a slow domain growth at low temperatures, where after certain time $t$ a characteristic domain size $L(t)$ is reached. The time evolution of quantities like the autocorrelation function will then present a crossover from dynamical processes characterized by length scales smaller than the already achieved domain size to processes at larger scales dominated by domain growth through the movement of domain walls. In this scenario, scaling arguments 11 leads to the following expected dependency of $C\left(t, t_{w}\right)$ :

$$
C\left(t, t_{w}\right) \propto c\left(L(t) / L\left(t_{w}\right)\right),
$$

at least for large values of $t$ and $t_{w}$.

Our results for $\delta>\delta_{c}$ are consistent with a $t / t_{w}$ scaling for large values of $\delta$, where the ferromagnetic short range interactions are dominant. This type of scaling, which is associated with an algebraic growth of the domain size $L(t) \propto t^{\psi}$, appears in the slow domain growth dynamics of disordered systems with a ferromagnetic ground state 9 - 11 .

Much more interesting is the logarithmic scaling form $\log (t) / \log \left(t_{w}\right)$ when $\delta<\delta_{c}$. This scaling is associated with a logarithmic time dependence of the domain size $L(t) \propto\left(\log t_{w}\right)^{\psi}$ predicted by an activated scenarid 14 in spin glasses, in which disorder and frustration generate active droplets excitations with a broad energy distribution.

In summary we have shown that the interplay between short- and long-range competitive interactions in an ordered system give rise to different kinds of aging at low temperatures. The different behaviours appear to be related to different domain dynamics as the relative strengths of the interactions are changed. In particular, for intermediate values of $\delta$, where the strengths are comparable, the competition between them generate a slow relaxation dynamic consistent with an activated scenario, like that proposed for spin glasses. This result suggests a possible relationship between the microscopic dynamic properties of this model and that of disordered frustrated systems. In this sense, it would be of interest to investigate, for instance, the possible existence of broad energy distributions of low-lying excitations in the present model. Works along these directions are in progress and would be published elsewhere.

Fruitful suggestions from Leticia F. Cugliandolo and Daniel Stariolo are aknowledged. This work was partially supported by grants from Consejo Nacional de Investigaciones Científicas y Técnicas CONICET (Argentina), Consejo Provincial de Investigaciones Científicas y Tecnológicas (Córdoba, Argentina) and Secretaría de Ciencia y Tecnología de la Universidad Nacional de Córdoba (Argentina).

E-mails: toloza@fis.uncor.edu, tamarit@fis.uncor.edu and cannas@fis.uncor.edu

* Member of the National Research Council, CONICET (Argentina)

${ }^{1}$ A. Kashuba and V. L. Pokrovsky, Phys. Rev. Lett. 70 (1993) 3155.

${ }^{2}$ A. B. MacIsaac, J. P. Whitehead, M. C. Robinson and K. De'Bell, Phys. Rev. B 51 (1995) 16033.

${ }^{3}$ L. C. Sampaio, M. P. de Albuquerque and F. S. de Menezes, Phys. Rev. B 54 (1996) 6465.

${ }^{4}$ D. Kivelson, S. A. Kivelson, X. Zhao, Z. Nussinov and G. Tarjus, Physica A 219 (1995) 129. 
${ }^{5}$ L. Chayes, V. J. Emery, S. A. Kivelson, Z. Nussinov and J. Tarjus, Physica A 225 (1996) 129.

${ }^{6}$ O. Zachar, S. A. Kivelson and V. J. Emery, Phys. Rev. B 57 (1998) 1422.

${ }^{7}$ L. P. Pryadko, S. Kivelson and D. W. Hone, cond-mat/9711129.

8 J-P. Bouchaud, L. F. Cugliandolo, J. Kurchan and M. Mezard, to appear in "Spin-glasses and random fields", A. P. Young

Ed. (World Scientific); also in cond-mat/9702070.

${ }^{9}$ T. J. Newman and A. J. Bray, J. Phys. A: Math. and Gen. 23 (1990) 4491.

${ }^{10}$ R. E. Blundell and A. J. Bray, J. Phys. A: Math. and Gen. 26, (1993) 5237.

${ }^{11}$ A. J. Bray, Advances in Physics 43 (1994) 357.

${ }^{12}$ L. Lundgren, P. Svedlindh, P. Nordblad and O. Beckman, Phys. Rev. Lett. 51 (1983) 911.

${ }^{13}$ H. Rieger, B. Steckemetz and M. Schreckenberg, Europhys. Lett. 27, (1994) 458.

${ }^{14}$ D. S. Fisher and D. A. Huse, Phys. Rev. B 38 (1988) 386.

FIG. 1. Snapshots of the time evolution of a $64 \times 64$ system with $\delta=4, T=0.5$ for different initial conditions. Figures (a), (b) and (c) correspond to an initial configuration with mean magnetization per site $m(0)=0$, for $t=1,10$ and 1000 Monte Carlo steps per site respectively. Figures (d), (e) and (f) correspond to an initial configuration with $m(0)=0.6$ and the same values of $t$.

FIG. 2. Snapshots of the time evolution of a $64 \times 64$ system with $\delta=2, T=0.5$ for different initial conditions. Figures (a), (b) and (c) correspond to an initial configuration with mean magnetization per site $m(0)=0$, for $t=1,10$ and 1000 Monte Carlo steps per site respectively. Figures (d), (e) and (f) correspond to an initial configuration with $m(0)=0.6$ and the same values of $t$.

FIG. 3. Autocorrelation function $C\left(t, t_{w}\right)$ vs the observation time $t$ at $T=0.5$, for $t_{w}=5^{2}$ (circles), $t_{w}=5^{3}$ (squares), $t_{w}=5^{4}$ (triangles) and $t_{w}=5^{5}$ (hexagons). (a) $\delta=-1$, inset: magnetization $m(t)$ (filled circles) and staggered magnetization $m_{s}(t)$ vs time. (b) $\delta=0.5$

FIG. 4. Autocorrelation function $C\left(t, t_{w}\right) v s$ the observation time $t$ for $t_{w}=5^{3}$ (circles), $t_{w}=5^{4}$ (squares), $t_{w}=5^{5}$ (triangles) and $t_{w}=5^{6}$ (hexagons), for different values of $\delta$ corresponding to the striped phase. Insets: magnetization $m(t) v s$ time starting from random $(m(0)=0$, open circles) and a fully magnetized $(m(0)=1$, filled circles) states.

FIG. 5. Data collapse of the curves shown in Fig.4 (the values of $t_{w}$ and the corresponding symbols are the same in both figures). 
a)

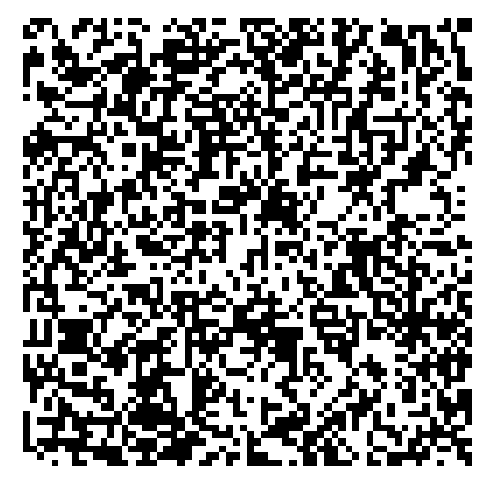

d)

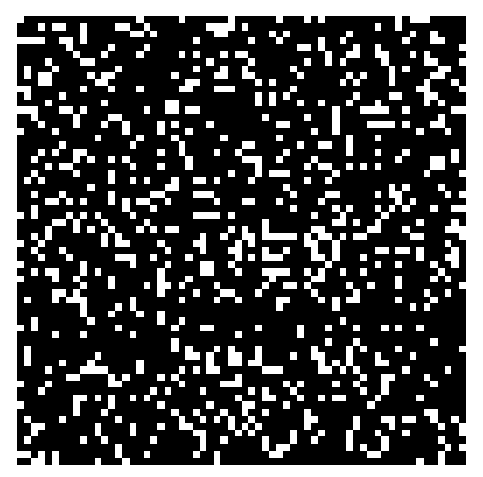

b)

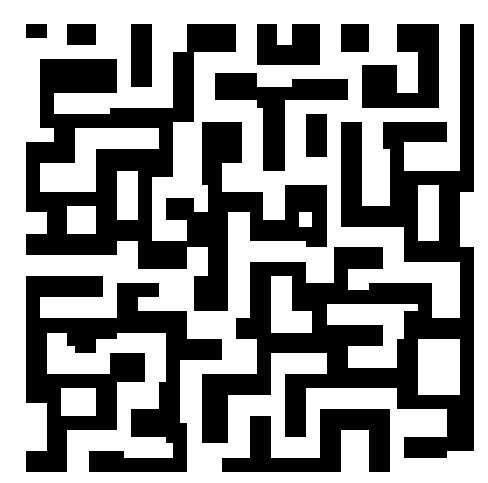

e)

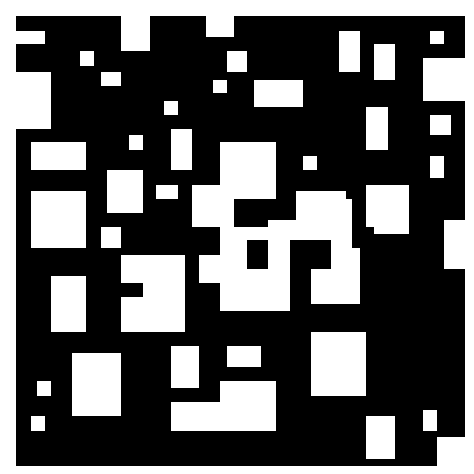

c)

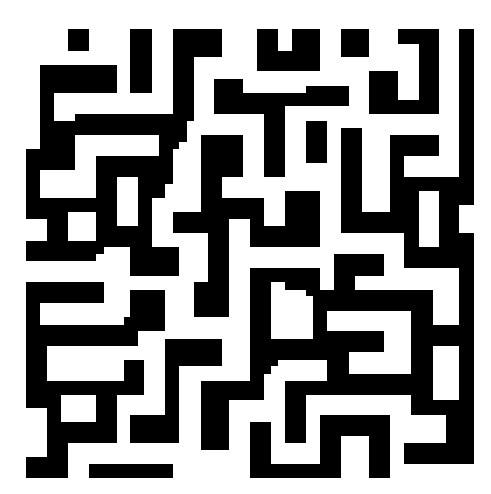

f)

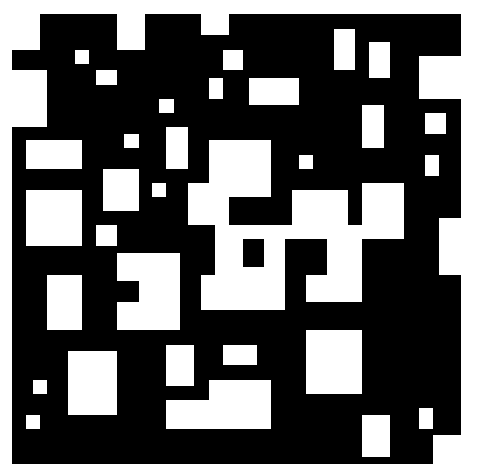

Figure 1 
a)

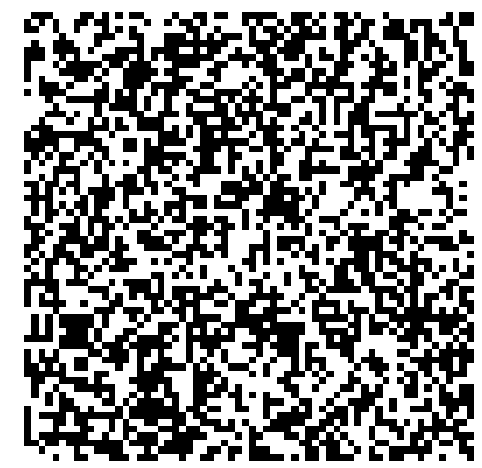

b)

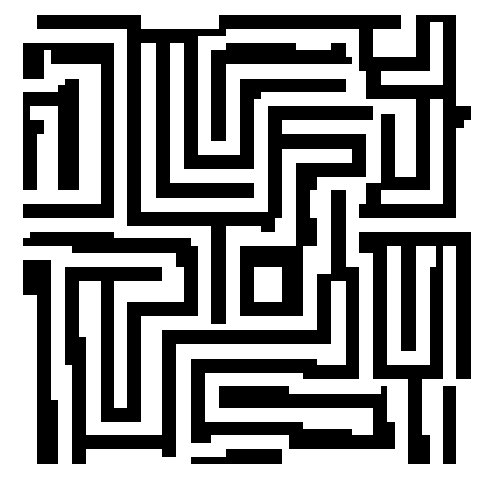

c)

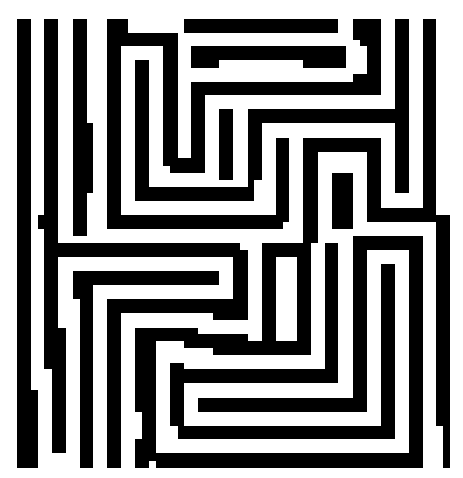

d)

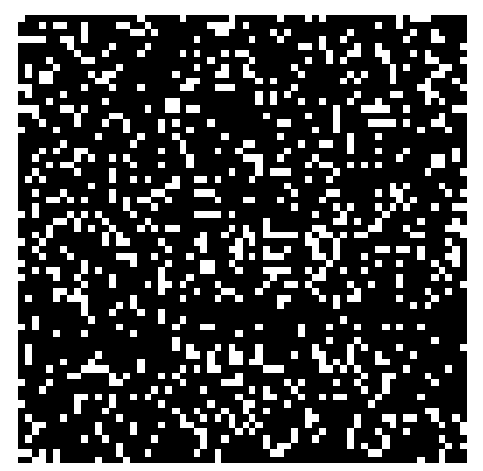

e)

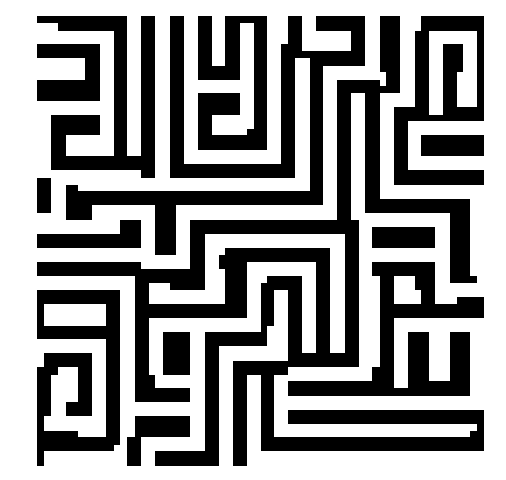

f)

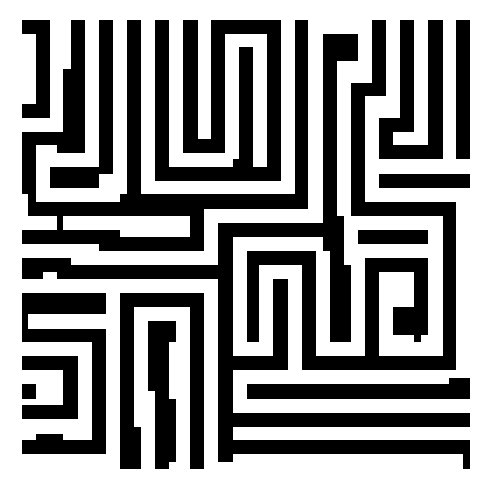

Figure 2 
a)

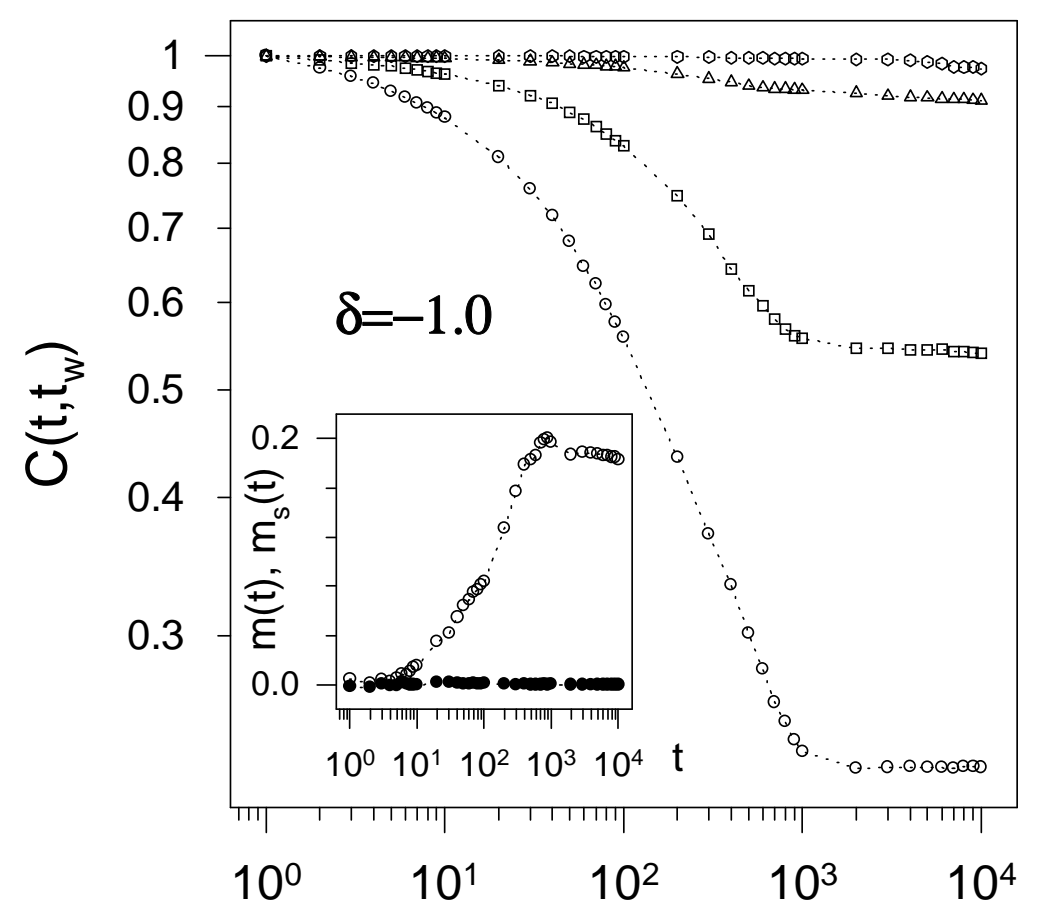

b)

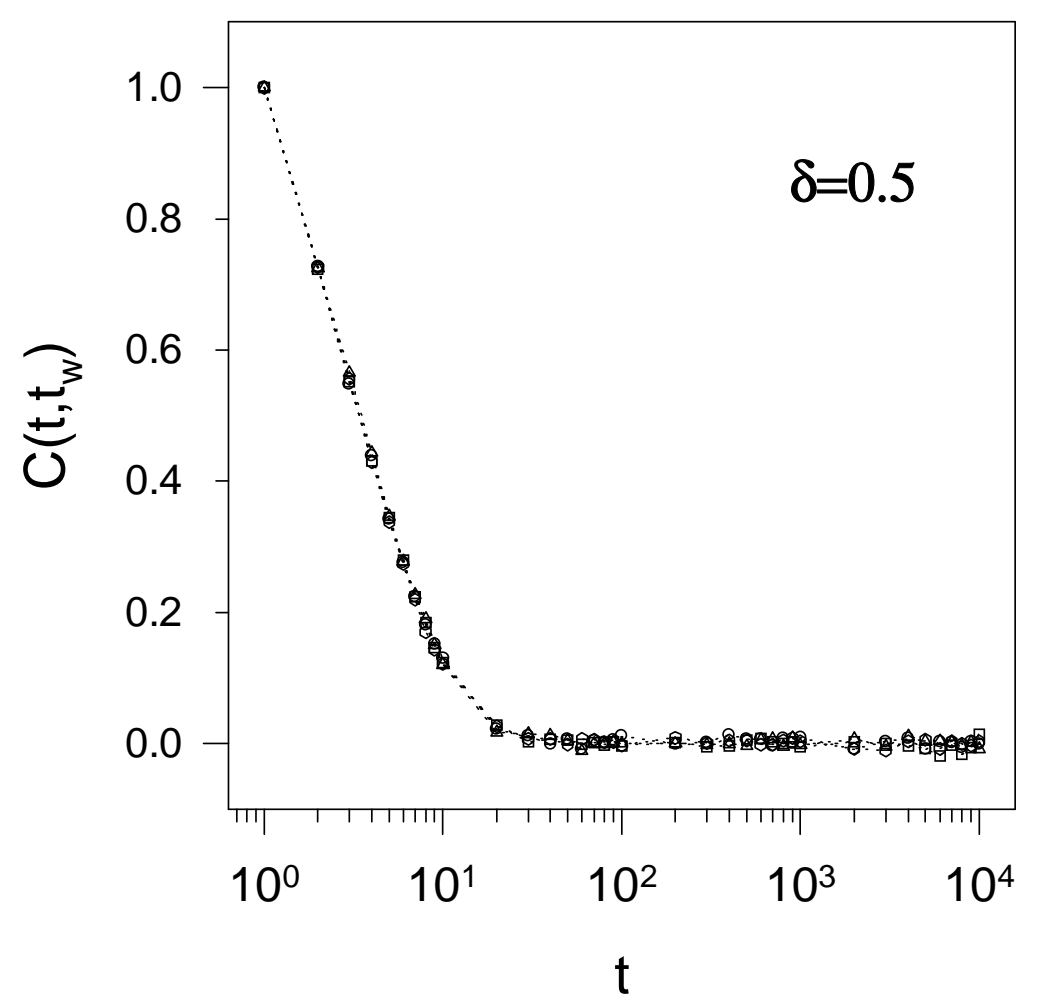

Figure 3 
a)

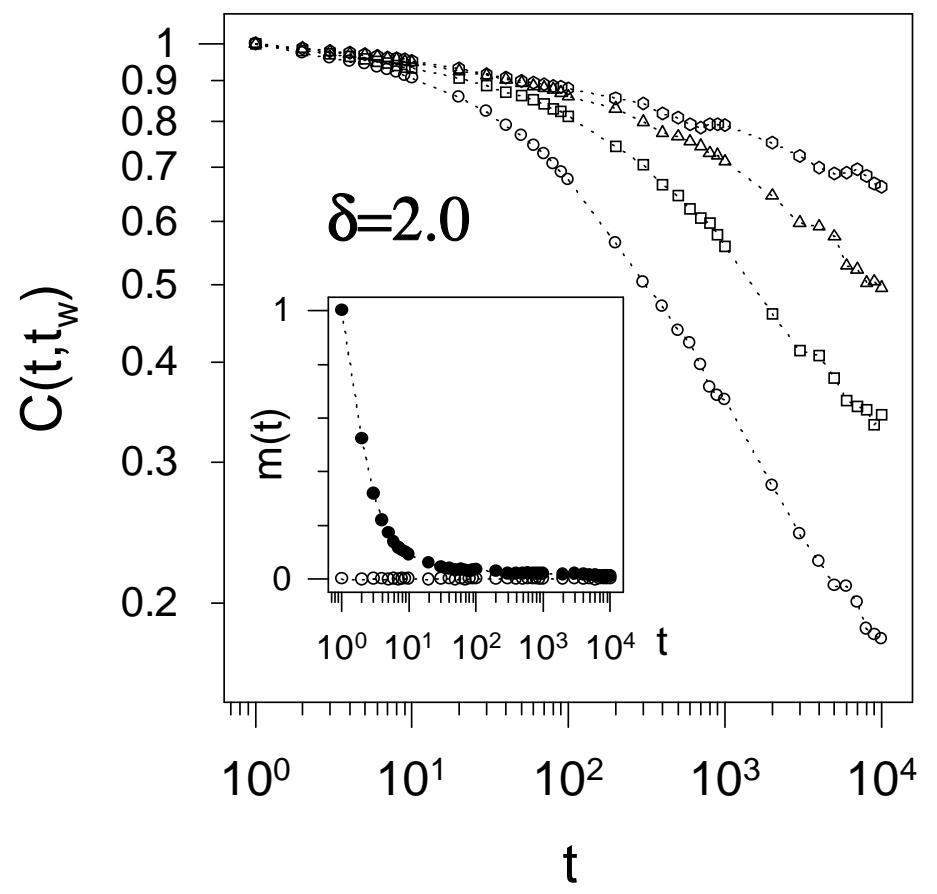

b)

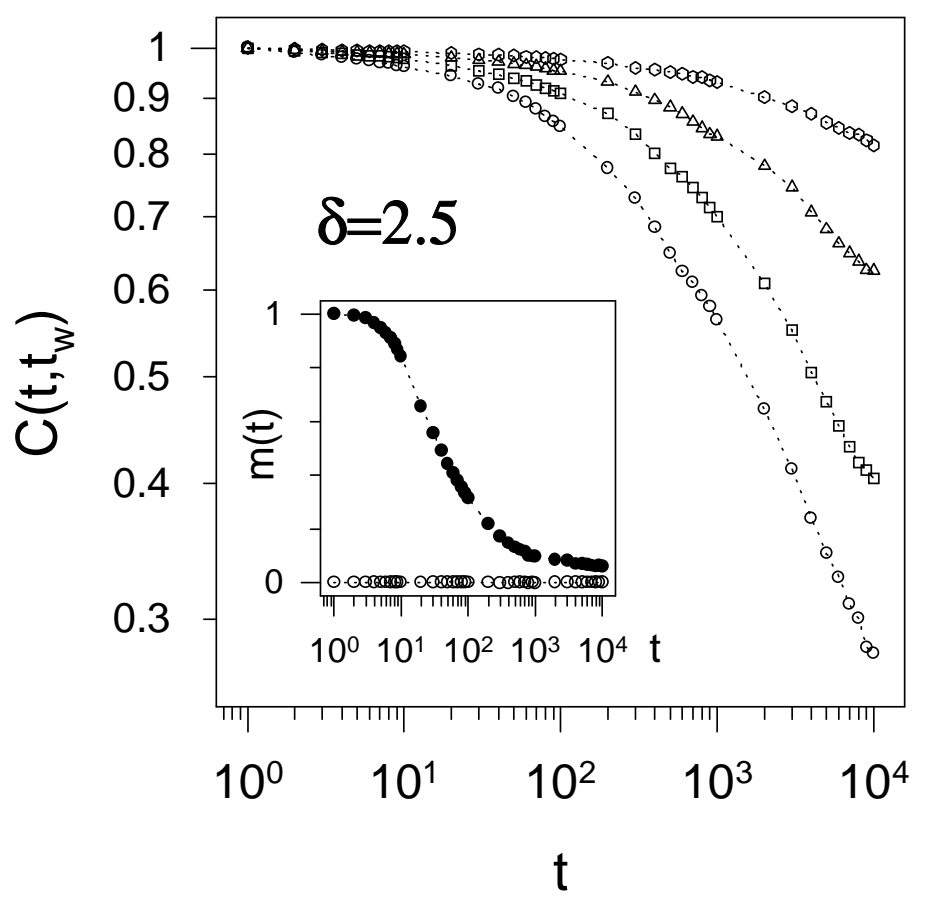

c)

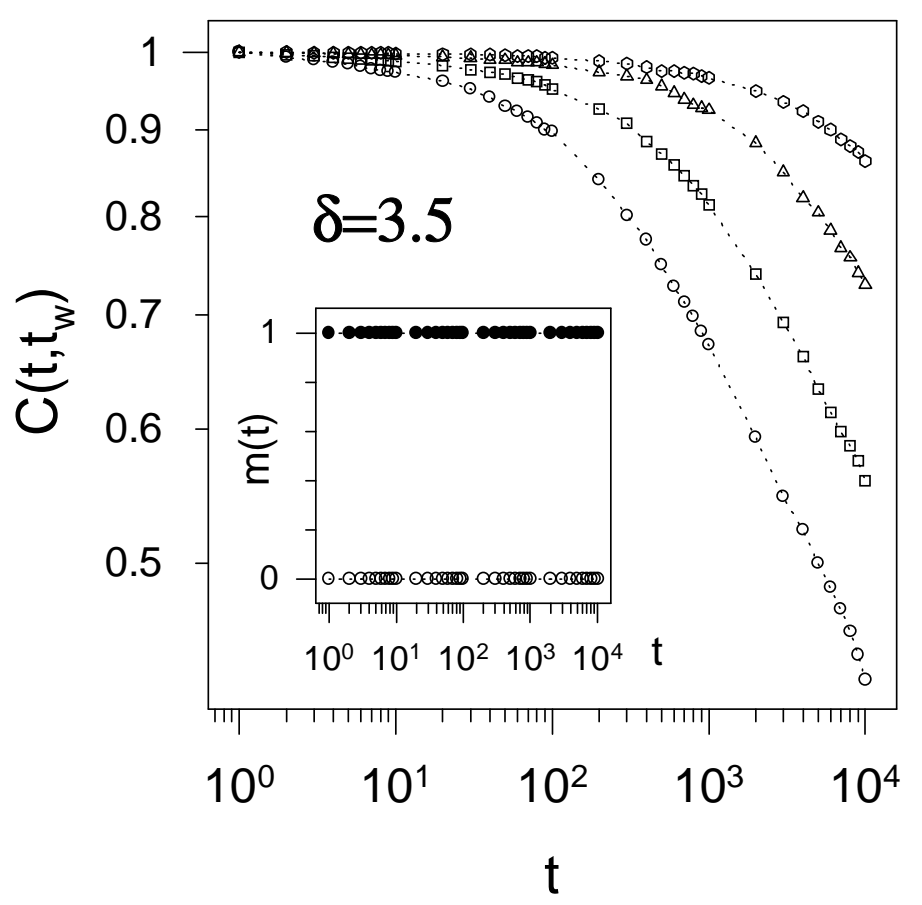

d)

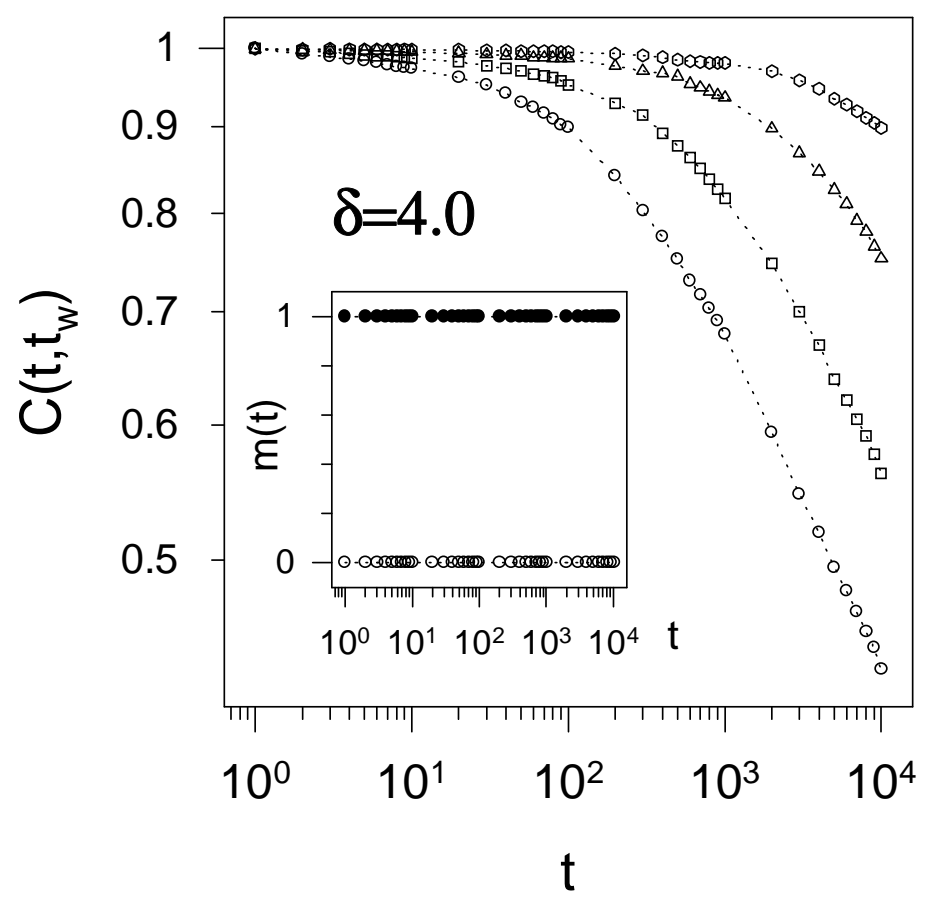

Figure 4 
a)

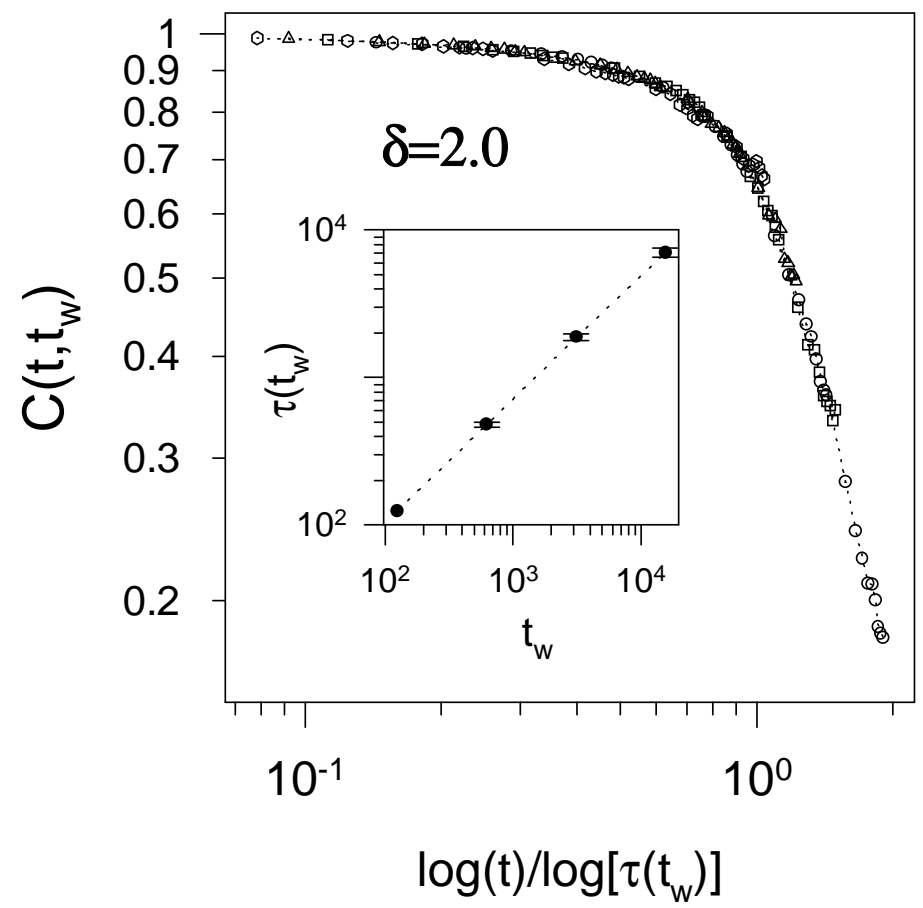

b)

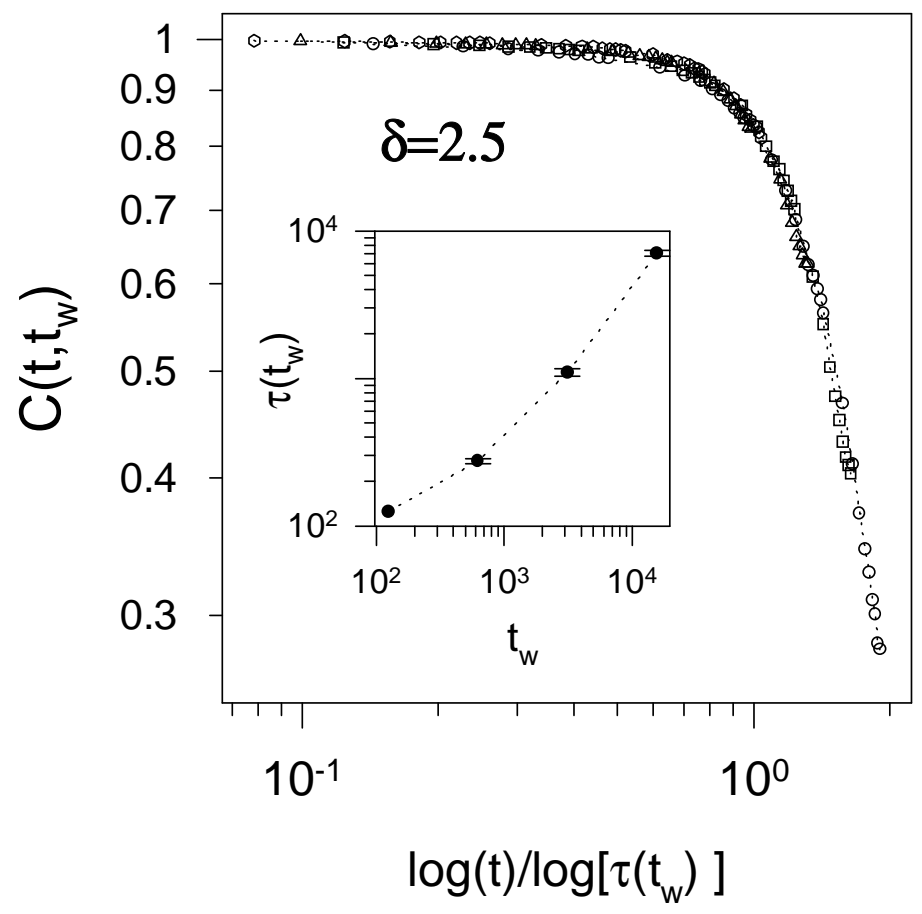

c)

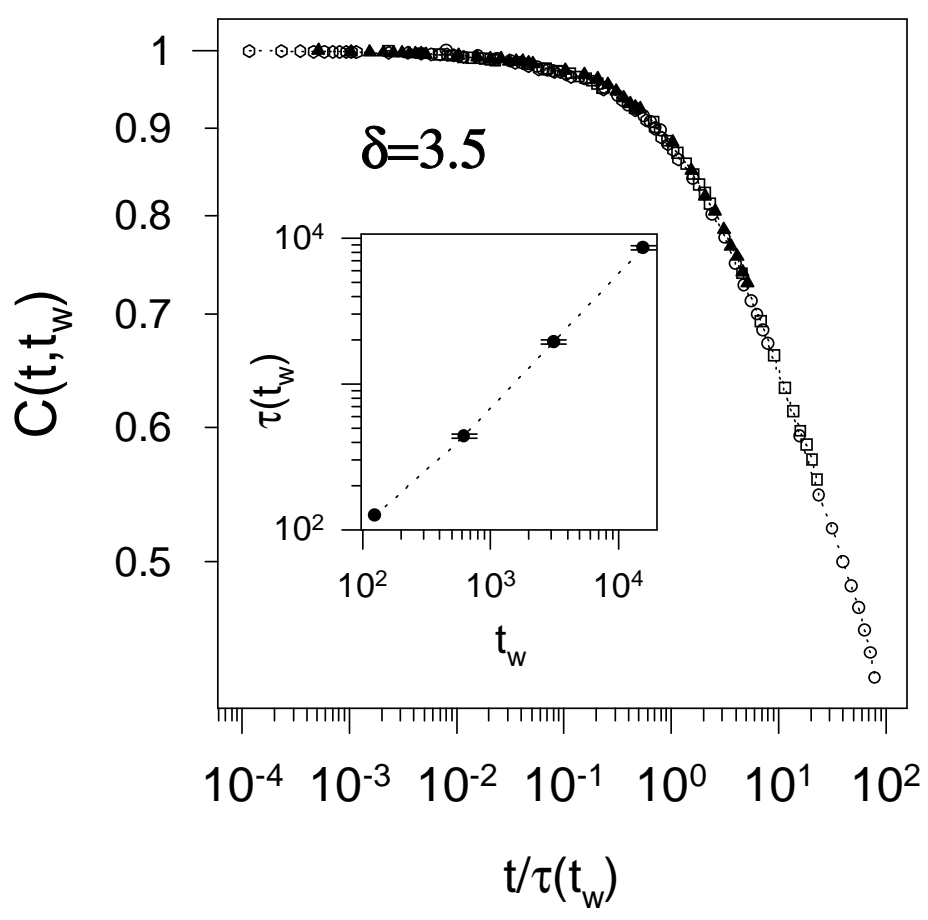

d)

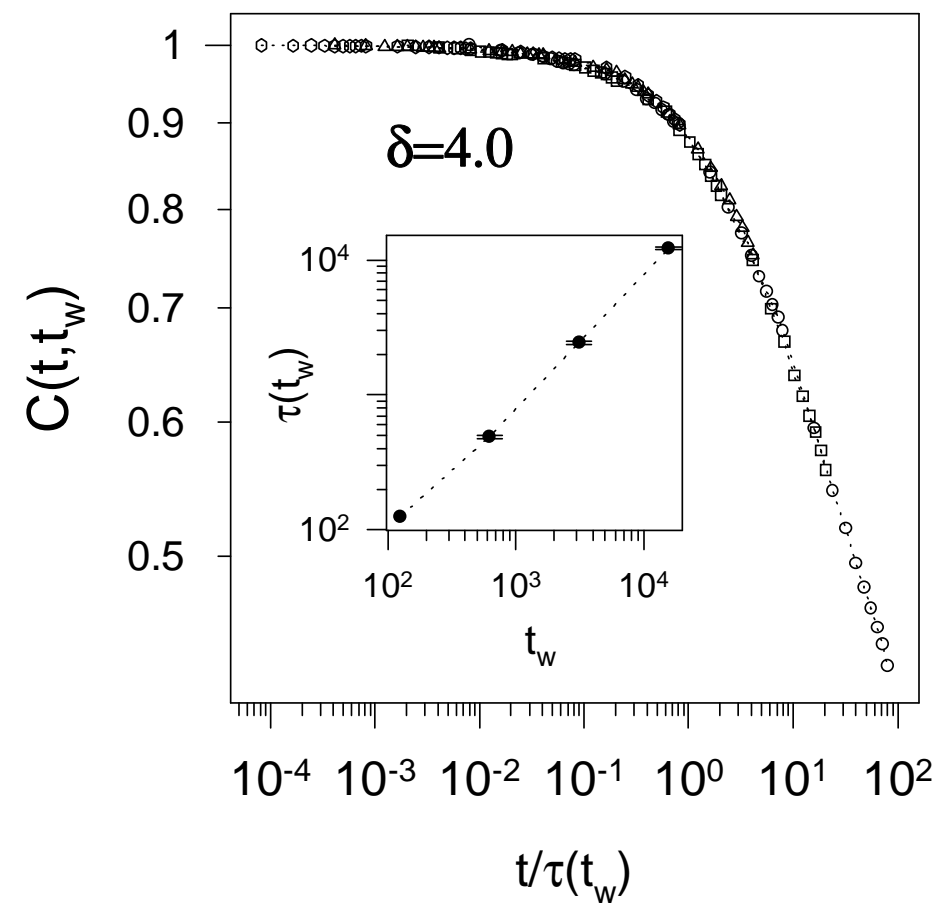

Figure 5 